\title{
A Comparison of Equality in Computer Algebra and Correctness in Mathematical Pedagogy
}

Russell Bradford, James Davenport \& Chris Sangwin

Universities of Bath, Bath (visiting Waterloo), Birmingham

27 June 2009 
Setting 


\section{Setting}

- A relatively traditional mathematics course, at, say first-year undergraduate level. 


\section{Setting}

- A relatively traditional mathematics course, at, say first-year undergraduate level.

- But Computer-Aided Assessment is in use. 


\section{Setting}

- A relatively traditional mathematics course, at, say first-year undergraduate level.

- But Computer-Aided Assessment is in use.

- One such example is WeBWorK, another is MapleTA. 


\section{Setting}

- A relatively traditional mathematics course, at, say first-year undergraduate level.

- But Computer-Aided Assessment is in use.

- One such example is WeBWorK, another is MapleTA.

- "Harness the power of technology to improve teaching and learning" [AMS Notices, June 2009]. [1] 


\section{Web-based Assessment and Testing Systems}

"Homework software has the potential to handle the grading of homework at a low cost. While this software has the limitation of requiring a concise answer - an algebraic expression or a multiple-choice response - it also has an important advantage over hand grading. Namely, if a students answer to a problem is wrong, the student learns of the mistake immediately and can be allowed to try the problem or a similar problem repeatedly until the right answer is obtained." [AMS] 


\section{Automatically generated problems}

Means automatically generated answers and marking schemes. 


\section{Automatically generated problems}

Means automatically generated answers and marking schemes.

- Parsing the student's answer (non-trivial — see next slide) 


\section{Automatically generated problems}

Means automatically generated answers and marking schemes.

- Parsing the student's answer (non-trivial - see next slide)

- Is the student's answer mathematically correct? 


\section{Automatically generated problems}

Means automatically generated answers and marking schemes.

- Parsing the student's answer (non-trivial - see next slide)

- Is the student's answer mathematically correct?

- Is the student's answer pedagogically correct? 


\section{Automatically generated problems}

Means automatically generated answers and marking schemes.

- Parsing the student's answer (non-trivial - see next slide)

- Is the student's answer mathematically correct?

- Is the student's answer pedagogically correct?

- So what mark does it get (assuming we are doing more than true/false marking)? 


\section{Automatically generated problems}

Means automatically generated answers and marking schemes.

- Parsing the student's answer (non-trivial - see next slide)

- Is the student's answer mathematically correct?

- Is the student's answer pedagogically correct?

- So what mark does it get (assuming we are doing more than true/false marking)? 


\section{Automatically generated problems}

Means automatically generated answers and marking schemes.

- Parsing the student's answer (non-trivial - see next slide)

- Is the student's answer mathematically correct?

- Is the student's answer pedagogically correct?

- So what mark does it get (assuming we are doing more than true/false marking)?

Marking other than true/false wa snot discussed by the MS, but seems important to us. 


\section{Typical computer aided assessment}

What is

$$
\frac{\mathrm{d} \sin ^{2} 2 x}{\mathrm{~d} x} ?
$$

$4 \sin (2 x)^{*} \cos (2 x)$

Your last answer was interpreted as:

$$
4 \cdot \sin (2 \cdot x) \cdot \cos (2 \cdot x)
$$

Correct answer, well done.

Your mark for this attempt is 1 .

Figure: STACK system [2] 


\section{Mathematical Correctness}

This is actually a non-trivial problem, even (especially?) with the resources of a computer algebra system behind us. 


\section{Mathematical Correctness}

This is actually a non-trivial problem, even (especially?) with the resources of a computer algebra system behind us.

- Can we prevent students from regurgitating the question? 


\section{Mathematical Correctness}

This is actually a non-trivial problem, even (especially?) with the resources of a computer algebra system behind us.

- Can we prevent students from regurgitating the question?

- Can we deal with 'smart alecs'? 


\section{Mathematical Correctness}

This is actually a non-trivial problem, even (especially?) with the resources of a computer algebra system behind us.

- Can we prevent students from regurgitating the question?

- Can we deal with 'smart alecs'?

- Probably no - a correct answer to "expand $(x+1)^{2 "}$ is

$$
x^{2}+\left(\max _{n \in \mathbb{N}} \exists x, y, z \in \mathbf{N}^{*} x^{n}+y^{n}=z^{n}\right) x+1 .
$$




\section{Mathematical Correctness}

This is actually a non-trivial problem, even (especially?) with the resources of a computer algebra system behind us.

- Can we prevent students from regurgitating the question?

- Can we deal with 'smart alecs'?

- Probably no - a correct answer to "expand $(x+1)^{2 "}$ is

$$
x^{2}+\left(\max _{n \in \mathbb{N}} \exists x, y, z \in \mathbf{N}^{*} x^{n}+y^{n}=z^{n}\right) x+1 .
$$

- How do we define "mathematical correctness" 


\section{Mathematical Correctness}

This is actually a non-trivial problem, even (especially?) with the resources of a computer algebra system behind us.

- Can we prevent students from regurgitating the question?

- Can we deal with 'smart alecs'?

- Probably no - a correct answer to "expand $(x+1)^{2 "}$ is

$$
x^{2}+\left(\max _{n \in \mathbb{N}} \exists x, y, z \in \mathbf{N}^{*} x^{n}+y^{n}=z^{n}\right) x+1 .
$$

- How do we define "mathematical correctness" 


\section{Mathematical Correctness}

This is actually a non-trivial problem, even (especially?) with the resources of a computer algebra system behind us.

- Can we prevent students from regurgitating the question?

- Can we deal with 'smart alecs'?

- Probably no - a correct answer to "expand $(x+1)^{2 "}$ is

$$
x^{2}+\left(\max _{n \in \mathbb{N}} \exists x, y, z \in \mathbf{N}^{*} x^{n}+y^{n}=z^{n}\right) x+1 .
$$

- How do we define "mathematical correctness"

WeBWork "if it's true at five points it's true" 


\section{Mathematical Correctness}

This is actually a non-trivial problem, even (especially?) with the resources of a computer algebra system behind us.

- Can we prevent students from regurgitating the question?

- Can we deal with 'smart alecs'?

- Probably no - a correct answer to "expand $(x+1)^{2 "}$ is

$$
x^{2}+\left(\max _{n \in \mathbf{N}} \exists x, y, z \in \mathbf{N}^{*} x^{n}+y^{n}=z^{n}\right) x+1 .
$$

- How do we define "mathematical correctness"

WeBWork "if it's true at five points it's true"

STACK Computer algebra - Maxima 


\section{Mathematical Correctness}

This is actually a non-trivial problem, even (especially?) with the resources of a computer algebra system behind us.

- Can we prevent students from regurgitating the question?

- Can we deal with 'smart alecs'?

- Probably no - a correct answer to "expand $(x+1)^{2 "}$ is

$$
x^{2}+\left(\max _{n \in \mathbf{N}} \exists x, y, z \in \mathbf{N}^{*} x^{n}+y^{n}=z^{n}\right) x+1 .
$$

- How do we define "mathematical correctness"

WeBWork "if it's true at five points it's true"

STACK Computer algebra - Maxima

Both have their drawbacks. 


\section{Pedagogical Correctness}

There's more to this than right/wrong 


\section{Pedagogical Correctness}

There's more to this than right/wrong

$$
\text { Table: Typical answers: } \frac{\mathrm{d} \sin ^{2} 2 x}{\mathrm{~d} x}
$$

$\begin{array}{lclr}\text { No. } & \text { Student's answer } & \text { C.A. } & \text { Score } \\ \text { 1. } & 4 \sin 2 x \cos 2 x & \mathrm{~T} & 1 \\ \text { 2. } & \frac{\mathrm{d} \sin ^{2} 2 x}{\mathrm{~d} x} & \mathrm{~T} & 0 \\ \text { 3. } & 2 \sin 2 x \cos 2 x & \mathrm{~F} & 0.7 \\ \text { 4. } & 2 \times 2 \sin 2 x \cos 2 x & \mathrm{~T} & 0.8 \\ \text { 5. } & 2 \sin 4 x & \mathrm{~T} ? & 1 \\ \text { 6. } & 2 \sin 2 x \cos 2 x+2 \sin 2 x \cos 2 x & \mathrm{~T} & 0.8 \\ \text { 7. } & x / 4-\sin (4 * x) / 8 & \mathrm{~F} & 0\end{array}$




\section{Pedagogical Correctness}

There's more to this than right/wrong

Table: Typical answers: $\frac{\mathrm{d} \sin ^{2} 2 x}{\mathrm{~d} x}$

$\begin{array}{lclr}\text { No. } & \text { Student's answer } & \text { C.A. } & \text { Score } \\ \text { 1. } & 4 \sin 2 x \cos 2 x & \mathrm{~T} & 1 \\ \text { 2. } & \frac{\mathrm{d} \sin ^{2} 2 x}{\mathrm{~d} x} & \mathrm{~T} & 0 \\ \text { 3. } & 2 \sin 2 x \cos 2 x & \mathrm{~F} & 0.7 \\ \text { 4. } & 2 \times 2 \sin 2 x \cos 2 x & \mathrm{~T} & 0.8 \\ \text { 5. } & 2 \sin 4 x & \mathrm{~T} ? & 1 \\ 6 . & 2 \sin 2 x \cos 2 x+2 \sin 2 x \cos 2 x & \mathrm{~T} & 0.8 \\ \text { 7. } & x / 4-\sin (4 * x) / 8 & \mathrm{~F} & 0\end{array}$

Note that both mathematically "right" and "wrong" answers got 0 , and a mathematically "wrong" answer still gets 70\%. 


\section{Pedagogical Correctness}

There's more to this than right/wrong

Table: Typical answers: $\frac{\mathrm{d} \sin ^{2} 2 x}{\mathrm{~d} x}$

$\begin{array}{lclr}\text { No. } & \text { Student's answer } & \text { C.A. } & \text { Score } \\ \text { 1. } & 4 \sin 2 x \cos 2 x & \mathrm{~T} & 1 \\ \text { 2. } & \frac{\mathrm{d} \sin ^{2} 2 x}{\mathrm{~d} x} & \mathrm{~T} & 0 \\ \text { 3. } & 2 \sin 2 x \cos 2 x & \mathrm{~F} & 0.7 \\ \text { 4. } & 2 \times 2 \sin 2 x \cos 2 x & \mathrm{~T} & 0.8 \\ \text { 5. } & 2 \sin 4 x & \mathrm{~T} ? & 1 \\ 6 . & 2 \sin 2 x \cos 2 x+2 \sin 2 x \cos 2 x & \mathrm{~T} & 0.8 \\ \text { 7. } & x / 4-\sin (4 * x) / 8 & \mathrm{~F} & 0\end{array}$

Note that both mathematically "right" and "wrong" answers got 0 , and a mathematically "wrong" answer still gets $70 \%$. We are not dealing with this second problem here! 
Marking a wrong answer 


\section{Marking a wrong answer}

This is not really within the scope of this talk: it falls more in the scope of "buggy rules" [3]. 


\section{Marking a wrong answer}

This is not really within the scope of this talk: it falls more in the scope of "buggy rules" [3].

- One might ask if it differs from the "true" answer by an additive constant 


\section{Marking a wrong answer}

This is not really within the scope of this talk: it falls more in the scope of "buggy rules" [3].

- One might ask if it differs from the "true" answer by an additive constant

- ... or a multiplicative constant (more likely for this sort of problem) 


\section{Marking a wrong answer}

This is not really within the scope of this talk: it falls more in the scope of "buggy rules" [3].

- One might ask if it differs from the "true" answer by an additive constant

- ... or a multiplicative constant (more likely for this sort of problem)

- or .... 


\section{What is (computer) algebra?}




\section{What is (computer) algebra?}

The Scratchpad/Axiom characterisation of computer algebra would be that it is working in a "sufficiently rich" order-sorted algebra, i.e. two expressions are equal if they are in the same congruence class, for a congruence generated by a "sufficiently rich" set of equations. For $\frac{\mathrm{d} \sin ^{2} 2 x}{\mathrm{~d} x}\left(\mathrm{D} \sin ^{2} 2 x\right)$, we would have rules like: 


\section{What is (computer) algebra?}

The Scratchpad/Axiom characterisation of computer algebra would be that it is working in a "sufficiently rich" order-sorted algebra, i.e. two expressions are equal if they are in the same congruence class, for a congruence generated by a "sufficiently rich" set of equations. For $\frac{\mathrm{d} \sin ^{2} 2 x}{\mathrm{~d} x}\left(\mathrm{D} \sin ^{2} 2 x\right)$, we would have rules like:

1. $\mathrm{a} * \mathrm{~b}=\mathrm{b} * \mathrm{a}$; 


\section{What is (computer) algebra?}

The Scratchpad/Axiom characterisation of computer algebra would be that it is working in a "sufficiently rich" order-sorted algebra, i.e. two expressions are equal if they are in the same congruence class, for a congruence generated by a "sufficiently rich" set of equations. For $\frac{\mathrm{d} \sin ^{2} 2 x}{\mathrm{~d} x}\left(\mathrm{D} \sin ^{2} 2 x\right)$, we would have rules like:

1. $\mathrm{a} * \mathrm{~b}=\mathrm{b} * \mathrm{a}$;

2. $\mathrm{m}+\mathrm{n}=m+n($ e.g. $2+3=5)$; 


\section{What is (computer) algebra?}

The Scratchpad/Axiom characterisation of computer algebra would be that it is working in a "sufficiently rich" order-sorted algebra, i.e. two expressions are equal if they are in the same congruence class, for a congruence generated by a "sufficiently rich" set of equations. For $\frac{\mathrm{d} \sin ^{2} 2 x}{\mathrm{~d} x}\left(\mathrm{D} \sin ^{2} 2 x\right)$, we would have rules like:

1. $\mathrm{a} * \mathrm{~b}=\mathrm{b} * \mathrm{a}$;

2. $\mathrm{m}+\mathrm{n}=m+n($ e.g. $2+3=5)$;

3. $\mathrm{a} * \mathrm{c}+\mathrm{b} * \mathrm{c}=(\mathrm{a}+\mathrm{b}) * \mathrm{c}$; 


\section{What is (computer) algebra?}

The Scratchpad/Axiom characterisation of computer algebra would be that it is working in a "sufficiently rich" order-sorted algebra, i.e. two expressions are equal if they are in the same congruence class, for a congruence generated by a "sufficiently rich" set of equations. For $\frac{\mathrm{d} \sin ^{2} 2 x}{\mathrm{~d} x}\left(\mathrm{D} \sin ^{2} 2 x\right)$, we would have rules like:

1. $\mathrm{a} * \mathrm{~b}=\mathrm{b} * \mathrm{a}$;

2. $\mathrm{m}+\mathrm{n}=m+n($ e.g. $2+3=5)$;

3. $\mathrm{a} * \mathrm{c}+\mathrm{b} * \mathrm{c}=(\mathrm{a}+\mathrm{b}) * \mathrm{c}$;

4. $\mathrm{x}^{\wedge} 1=\mathrm{x}$; 


\section{What is (computer) algebra?}

The Scratchpad/Axiom characterisation of computer algebra would be that it is working in a "sufficiently rich" order-sorted algebra, i.e. two expressions are equal if they are in the same congruence class, for a congruence generated by a "sufficiently rich" set of equations. For $\frac{\mathrm{d} \sin ^{2} 2 x}{\mathrm{~d} x}\left(\mathrm{D} \sin ^{2} 2 x\right)$, we would have rules like:

1. $\mathrm{a} * \mathrm{~b}=\mathrm{b} * \mathrm{a}$;

2. $\mathrm{m}+\mathrm{n}=m+n($ e.g. $2+3=5)$;

3. $\mathrm{a} * \mathrm{c}+\mathrm{b} * \mathrm{c}=(\mathrm{a}+\mathrm{b}) * \mathrm{c}$;

4. $\mathrm{x}^{\wedge} 1=\mathrm{x}$;

5. $D\left(a^{\wedge} n\right)=n * a^{\wedge}(n-1) * D(a)$; 


\section{What is (computer) algebra?}

The Scratchpad/Axiom characterisation of computer algebra would be that it is working in a "sufficiently rich" order-sorted algebra, i.e. two expressions are equal if they are in the same congruence class, for a congruence generated by a "sufficiently rich" set of equations. For $\frac{\mathrm{d} \sin ^{2} 2 x}{\mathrm{~d} x}\left(\mathrm{D} \sin ^{2} 2 x\right)$, we would have rules like:

1. $\mathrm{a} * \mathrm{~b}=\mathrm{b} * \mathrm{a}$;

2. $\mathrm{m}+\mathrm{n}=m+n($ e.g. $2+3=5)$;

3. $\mathrm{a} * \mathrm{c}+\mathrm{b} * \mathrm{c}=(\mathrm{a}+\mathrm{b}) * \mathrm{c}$;

4. $\mathrm{x}^{\wedge} 1=\mathrm{x}$;

5. $D\left(a^{\wedge} n\right)=n * a^{\wedge}(n-1) * D(a)$;

6. $D(\sin (a))=\cos (a) * D(a)$. 


\section{What is (computer) algebra?}

The Scratchpad/Axiom characterisation of computer algebra would be that it is working in a "sufficiently rich" order-sorted algebra, i.e. two expressions are equal if they are in the same congruence class, for a congruence generated by a "sufficiently rich" set of equations. For $\frac{\mathrm{d} \sin ^{2} 2 x}{\mathrm{~d} x}\left(\mathrm{D} \sin ^{2} 2 x\right)$, we would have rules like:

1. $\mathrm{a} * \mathrm{~b}=\mathrm{b} * \mathrm{a}$;

2. $\mathrm{m}+\mathrm{n}=m+n($ e.g. $2+3=5)$;

3. $\mathrm{a} * \mathrm{c}+\mathrm{b} * \mathrm{c}=(\mathrm{a}+\mathrm{b}) * \mathrm{c}$;

4. $\mathrm{x}^{\wedge} 1=\mathrm{x}$;

5. $D\left(a^{\wedge} n\right)=n * a^{\wedge}(n-1) * D(a)$;

6. $D(\sin (a))=\cos (a) * D(a)$. 


\section{What is (computer) algebra?}

The Scratchpad/Axiom characterisation of computer algebra would be that it is working in a "sufficiently rich" order-sorted algebra, i.e. two expressions are equal if they are in the same congruence class, for a congruence generated by a "sufficiently rich" set of equations. For $\frac{\mathrm{d} \sin ^{2} 2 x}{\mathrm{~d} x}\left(\mathrm{D} \sin ^{2} 2 x\right)$, we would have rules like:

1. $\mathrm{a} * \mathrm{~b}=\mathrm{b} * \mathrm{a}$;

2. $\mathrm{m}+\mathrm{n}=m+n($ e.g. $2+3=5)$;

3. $\mathrm{a} * \mathrm{c}+\mathrm{b} * \mathrm{c}=(\mathrm{a}+\mathrm{b}) * \mathrm{c}$;

4. $\mathrm{x}^{\wedge} 1=\mathrm{x}$;

5. $D\left(a^{\wedge} \mathrm{n}\right)=\mathrm{n} * \mathrm{a}^{\wedge}(\mathrm{n}-1) * \mathrm{D}(\mathrm{a})$;

6. $D(\sin (a))=\cos (a) * D(a)$.

Note that it need not be implemented this way. 


\section{Utility of this model}




\section{Utility of this model}

In this model, it is indeed true that $\frac{\mathrm{d} \sin ^{2} 2 x}{\mathrm{~d} x}=4 \sin 2 x \cos 2 x$ 


\section{Utility of this model}

In this model, it is indeed true that $\frac{\mathrm{d} \sin ^{2} 2 x}{\mathrm{~d} x}=4 \sin 2 x \cos 2 x$ We can now divide the rules into three categories, which we call underlying, 


\section{Utility of this model}

In this model, it is indeed true that $\frac{\mathrm{d} \sin ^{2} 2 x}{\mathrm{~d} x}=4 \sin 2 x \cos 2 x$ We can now divide the rules into three categories, which we call underlying, venial (failure to use effectively costs fractions of a point) 


\section{Utility of this model}

In this model, it is indeed true that $\frac{\mathrm{d} \sin ^{2} 2 x}{\mathrm{~d} x}=4 \sin 2 x \cos 2 x$ We can now divide the rules into three categories, which we call underlying, venial (failure to use effectively costs fractions of a point) and fatal (needing this to get the "right" answer is fatal). 


\section{Utility of this model}

In this model, it is indeed true that $\frac{\mathrm{d} \sin ^{2} 2 x}{\mathrm{~d} x}=4 \sin 2 x \cos 2 x$ We can now divide the rules into three categories, which we call underlying, venial (failure to use effectively costs fractions of a point) and fatal (needing this to get the "right" answer is fatal). Our equations are then categorised as follows.

U1 $\mathrm{a} * \mathrm{~b}=\mathrm{b} * \mathrm{a}$; 


\section{Utility of this model}

In this model, it is indeed true that $\frac{\mathrm{d} \sin ^{2} 2 x}{\mathrm{~d} x}=4 \sin 2 x \cos 2 x$ We can now divide the rules into three categories, which we call underlying, venial (failure to use effectively costs fractions of a point) and fatal (needing this to get the "right" answer is fatal). Our equations are then categorised as follows.

U1 $\mathrm{a} * \mathrm{~b}=\mathrm{b} * \mathrm{a}$;

$\mathrm{V} 2 \mathrm{~m}+\mathrm{n}=m+n($ e.g. $2+3=5)($ and -$)$; 


\section{Utility of this model}

In this model, it is indeed true that $\frac{\mathrm{d} \sin ^{2} 2 x}{\mathrm{~d} x}=4 \sin 2 x \cos 2 x$ We can now divide the rules into three categories, which we call underlying, venial (failure to use effectively costs fractions of a point) and fatal (needing this to get the "right" answer is fatal). Our equations are then categorised as follows.

U1 $\mathrm{a} * \mathrm{~b}=\mathrm{b} * \mathrm{a}$;

$\mathrm{V} 2 \mathrm{~m}+\mathrm{n}=m+n($ e.g. $2+3=5)($ and -$)$;

V3 $\mathrm{a} * \mathrm{c}+\mathrm{b} * \mathrm{c}=(\mathrm{a}+\mathrm{b}) * \mathrm{c}$; 


\section{Utility of this model}

In this model, it is indeed true that $\frac{\mathrm{d} \sin ^{2} 2 x}{\mathrm{~d} x}=4 \sin 2 x \cos 2 x$ We can now divide the rules into three categories, which we call underlying, venial (failure to use effectively costs fractions of a point) and fatal (needing this to get the "right" answer is fatal). Our equations are then categorised as follows.

U1 $\mathrm{a} * \mathrm{~b}=\mathrm{b} * \mathrm{a}$;

$\mathrm{V} 2 \mathrm{~m}+\mathrm{n}=m+n($ e.g. $2+3=5)($ and -$)$;

V3 $\mathrm{a} * \mathrm{c}+\mathrm{b} * \mathrm{c}=(\mathrm{a}+\mathrm{b}) * \mathrm{c}$;

V4 $\mathrm{x}^{\wedge} 1=\mathrm{x}$; 


\section{Utility of this model}

In this model, it is indeed true that $\frac{\mathrm{d} \sin ^{2} 2 x}{\mathrm{~d} x}=4 \sin 2 x \cos 2 x$ We can now divide the rules into three categories, which we call underlying, venial (failure to use effectively costs fractions of a point) and fatal (needing this to get the "right" answer is fatal). Our equations are then categorised as follows.

U1 $\mathrm{a} * \mathrm{~b}=\mathrm{b} * \mathrm{a}$;

$\mathrm{V} 2 \mathrm{~m}+\mathrm{n}=m+n($ e.g. $2+3=5)($ and -$)$;

V3 $\mathrm{a} * \mathrm{c}+\mathrm{b} * \mathrm{c}=(\mathrm{a}+\mathrm{b}) * \mathrm{c}$;

V4 $\mathrm{x}^{\wedge} 1=\mathrm{x}$;

F1 $D\left(a^{\wedge} \mathrm{n}\right)=\mathrm{n} * \mathrm{a}^{\wedge}(\mathrm{n}-1) * \mathrm{D}(\mathrm{a})$; 


\section{Utility of this model}

In this model, it is indeed true that $\frac{\mathrm{d} \sin ^{2} 2 x}{\mathrm{~d} x}=4 \sin 2 x \cos 2 x$ We can now divide the rules into three categories, which we call underlying, venial (failure to use effectively costs fractions of a point) and fatal (needing this to get the "right" answer is fatal). Our equations are then categorised as follows.

U1 $\mathrm{a} * \mathrm{~b}=\mathrm{b} * \mathrm{a}$;

$\mathrm{V} 2 \mathrm{~m}+\mathrm{n}=m+n($ e.g. $2+3=5)($ and -$)$;

V3 $\mathrm{a} * \mathrm{c}+\mathrm{b} * \mathrm{c}=(\mathrm{a}+\mathrm{b}) * \mathrm{c}$;

V4 $\mathrm{x}^{\wedge} 1=\mathrm{x}$;

F1 $D\left(a^{\wedge} \mathrm{n}\right)=\mathrm{n} * \mathrm{a}^{\wedge}(\mathrm{n}-1) * \mathrm{D}(\mathrm{a})$;

F2 $D(\sin (a))=\cos (a) * D(a)$. 


\section{Answers analysed}

Let $\mathcal{U}$ be the set of underlying equations, $\mathcal{V}$ be the underlying and venial ones, and $\mathcal{F}$ the set of them all. 


\section{Answers analysed}

Let $\mathcal{U}$ be the set of underlying equations, $\mathcal{V}$ be the underlying and venial ones, and $\mathcal{F}$ the set of them all.

Table: Analysed answers: $\frac{\mathrm{d} \sin ^{2} 2 x}{\mathrm{~d} x}=4 \cos 2 x \sin 2 x$

$\begin{array}{lclr}\text { No. } & \text { Student's answer } & \text { relation } & \text { Score } \\ \text { 1. } & 4 \sin 2 x \cos 2 x & \equiv \mathcal{U} & 1 \\ \text { 2. } & \frac{\mathrm{d} \sin ^{2} 2 x}{\mathrm{~d} x} & \equiv \mathcal{F} & 0 \\ \text { 3. } & 2 \sin 2 x \cos 2 x & \mathrm{~F} & \text { buggy } \\ \text { 4. } & 2 \times 2 \sin 2 x \cos 2 x & \equiv \mathcal{V} & 0.8 \\ \text { 5. } & 2 \sin 4 x & \text { none } & 0 \\ \text { 6. } & 2 \sin 2 x \cos 2 x+2 \sin 2 x \cos 2 x & \equiv \mathcal{V} & 0.8 \\ \text { 7. } & x / 4-\sin (4 * x) / 8 & \text { none } & 0\end{array}$




\section{Answers analysed}

Let $\mathcal{U}$ be the set of underlying equations, $\mathcal{V}$ be the underlying and venial ones, and $\mathcal{F}$ the set of them all.

Table: Analysed answers: $\frac{\mathrm{d} \sin ^{2} 2 x}{\mathrm{~d} x}=4 \cos 2 x \sin 2 x$

$\begin{array}{lclr}\text { No. } & \text { Student's answer } & \text { relation } & \text { Score } \\ \text { 1. } & 4 \sin 2 x \cos 2 x & \equiv \mathcal{U} & 1 \\ \text { 2. } & \frac{\mathrm{d} \sin ^{2} 2 x}{\mathrm{~d} x} & \equiv \mathcal{F} & 0 \\ \text { 3. } & 2 \sin 2 x \cos 2 x & \mathrm{~F} & \text { buggy } \\ \text { 4. } & 2 \times 2 \sin 2 x \cos 2 x & \equiv \mathcal{V} & 0.8 \\ \text { 5. } & 2 \sin 4 x & \text { none } & 0 \\ \text { 6. } & 2 \sin 2 x \cos 2 x+2 \sin 2 x \cos 2 x & \equiv \mathcal{V} & 0.8 \\ \text { 7. } & x / 4-\sin (4 * x) / 8 & \text { none } & 0\end{array}$

Note that answer 5 is marked wrong, since trigonometric contraction is not one of our rules. It probably should be, but we need a digression. 


\section{What is the "right" answer}

Assuming we do not know about trigonometric contraction, most people would say $4 \sin 2 x \cos 2 x$ (or $4 \cos 2 x \sin 2 x$, which is equivalent under $\mathcal{U}$ ). 


\section{What is the "right" answer}

Assuming we do not know about trigonometric contraction, most people would say $4 \sin 2 x \cos 2 x$ (or $4 \cos 2 x \sin 2 x$, which is equivalent under $\mathcal{U})$. But mathematically this is

$$
\sin 2 x \cos 2 x+3 \cos 2 x \sin 2 x
$$

(and many other expressions). 


\section{What is the "right" answer}

Assuming we do not know about trigonometric contraction, most people would say $4 \sin 2 x \cos 2 x$ (or $4 \cos 2 x \sin 2 x$, which is equivalent under $\mathcal{U})$. But mathematically this is

$$
\sin 2 x \cos 2 x+3 \cos 2 x \sin 2 x
$$

(and many other expressions). Of course, we really want the "simplest" answer. What does "simplify" mean? 


\section{What is the "right" answer}

Assuming we do not know about trigonometric contraction, most people would say $4 \sin 2 x \cos 2 x$ (or $4 \cos 2 x \sin 2 x$, which is equivalent under $\mathcal{U}$ ). But mathematically this is

$$
\sin 2 x \cos 2 x+3 \cos 2 x \sin 2 x
$$

(and many other expressions). Of course, we really want the "simplest" answer. What does "simplify" mean?

- "simplify by removing brackets and collecting like terms". 


\section{What is the "right" answer}

Assuming we do not know about trigonometric contraction, most people would say $4 \sin 2 x \cos 2 x$ (or $4 \cos 2 x \sin 2 x$, which is equivalent under $\mathcal{U}$ ). But mathematically this is

$$
\sin 2 x \cos 2 x+3 \cos 2 x \sin 2 x
$$

(and many other expressions). Of course, we really want the "simplest" answer. What does "simplify" mean?

- "simplify by removing brackets and collecting like terms".

- "factor and cancel like terms" (in the same book!) 


\section{What is the "right" answer}

Assuming we do not know about trigonometric contraction, most people would say $4 \sin 2 x \cos 2 x$ (or $4 \cos 2 x \sin 2 x$, which is equivalent under $\mathcal{U}$ ). But mathematically this is

$$
\sin 2 x \cos 2 x+3 \cos 2 x \sin 2 x
$$

(and many other expressions). Of course, we really want the "simplest" answer. What does "simplify" mean?

- "simplify by removing brackets and collecting like terms".

- "factor and cancel like terms" (in the same book!)

- "do what I've just shown you" (often). 


\section{What is the "right" answer}

Assuming we do not know about trigonometric contraction, most people would say $4 \sin 2 x \cos 2 x$ (or $4 \cos 2 x \sin 2 x$, which is equivalent under $\mathcal{U}$ ). But mathematically this is

$$
\sin 2 x \cos 2 x+3 \cos 2 x \sin 2 x
$$

(and many other expressions). Of course, we really want the "simplest" answer. What does "simplify" mean?

- "simplify by removing brackets and collecting like terms".

- "factor and cancel like terms" (in the same book!)

- "do what l've just shown you" (often).

- "Give me the answer I want" (Classes préparatoires professors to JHD). 


\section{What is the "right" answer}

Assuming we do not know about trigonometric contraction, most people would say $4 \sin 2 x \cos 2 x$ (or $4 \cos 2 x \sin 2 x$, which is equivalent under $\mathcal{U}$ ). But mathematically this is

$$
\sin 2 x \cos 2 x+3 \cos 2 x \sin 2 x
$$

(and many other expressions). Of course, we really want the "simplest" answer. What does "simplify" mean?

- "simplify by removing brackets and collecting like terms".

- "factor and cancel like terms" (in the same book!)

- "do what l've just shown you" (often).

- "Give me the answer I want" (Classes préparatoires professors to JHD).

- [Carette 2004] "The/A shortest equivalent expression". 


\section{What is the "right" answer}

Assuming we do not know about trigonometric contraction, most people would say $4 \sin 2 x \cos 2 x$ (or $4 \cos 2 x \sin 2 x$, which is equivalent under $\mathcal{U}$ ). But mathematically this is

$$
\sin 2 x \cos 2 x+3 \cos 2 x \sin 2 x
$$

(and many other expressions). Of course, we really want the "simplest" answer. What does "simplify" mean?

- "simplify by removing brackets and collecting like terms".

- "factor and cancel like terms" (in the same book!)

- "do what l've just shown you" (often).

- "Give me the answer I want" (Classes préparatoires professors to JHD).

- [Carette 2004] "The/A shortest equivalent expression". 


\section{What is the "right" answer}

Assuming we do not know about trigonometric contraction, most people would say $4 \sin 2 x \cos 2 x$ (or $4 \cos 2 x \sin 2 x$, which is equivalent under $\mathcal{U}$ ). But mathematically this is

$$
\sin 2 x \cos 2 x+3 \cos 2 x \sin 2 x
$$

(and many other expressions). Of course, we really want the "simplest" answer. What does "simplify" mean?

- "simplify by removing brackets and collecting like terms".

- "factor and cancel like terms" (in the same book!)

- "do what l've just shown you" (often).

- "Give me the answer I want" (Classes préparatoires professors to JHD).

- [Carette 2004] "The/A shortest equivalent expression".

"The right answer" is "a shortest expression under $\equiv \mathcal{F}$ ". 


\section{What is the "right" answer}

Assuming we do not know about trigonometric contraction, most people would say $4 \sin 2 x \cos 2 x$ (or $4 \cos 2 x \sin 2 x$, which is equivalent under $\mathcal{U}$ ). But mathematically this is

$$
\sin 2 x \cos 2 x+3 \cos 2 x \sin 2 x
$$

(and many other expressions). Of course, we really want the "simplest" answer. What does "simplify" mean?

- "simplify by removing brackets and collecting like terms".

- "factor and cancel like terms" (in the same book!)

- "do what l've just shown you" (often).

- "Give me the answer I want" (Classes préparatoires professors to JHD).

- [Carette 2004] "The/A shortest equivalent expression".

"The right answer" is "a shortest expression under $\equiv \mathcal{F}$ ". It had better be the case that only $\mathcal{U}$ can produce equivalent expressions of the same length. 


\section{Answers re-analysed}

Add various fraction-simplifying rules to $\mathcal{V}$, and

U2 $\sin \mathrm{a} * \cos \mathrm{a}=\frac{1}{2} \sin 2 * \mathrm{a}$. 


\section{Answers re-analysed}

Add various fraction-simplifying rules to $\mathcal{V}$, and

U2 $\sin \mathrm{a} * \cos \mathrm{a}=\frac{1}{2} \sin 2 * \mathrm{a}$. 


\section{Answers re-analysed}

Add various fraction-simplifying rules to $\mathcal{V}$, and

$$
\text { U2 } \sin a * \cos a=\frac{1}{2} \sin 2 * a \text {. }
$$

Table: Re-analysed answers: $\frac{\mathrm{d} \sin ^{2} 2 x}{\mathrm{~d} x}=2 \sin 4 x$

$\begin{array}{lclr}\text { No. } & \text { Student's answer } & \text { relation } & \text { Score } \\ \text { 1. } & 4 \sin 2 x \cos 2 x & \equiv \mathcal{U} & 1 \\ \text { 2. } & \frac{\mathrm{d} \sin ^{2} 2 x}{\mathrm{~d} x} & \equiv \mathcal{F} & 0 \\ \text { 3. } & 2 \sin 2 x \cos 2 x & \mathrm{~F} & \text { buggy } \\ \text { 4. } & 2 \times 2 \sin 2 x \cos 2 x & \equiv \mathcal{V} & 0.8 \\ \text { 5. } & 2 \sin 4 x & = & 1 \\ 6 . & 2 \sin 2 x \cos 2 x+2 \sin 2 x \cos 2 x & \equiv \mathcal{V} & 0.8 \\ \text { 7. } & x / 4-\sin (4 * x) / 8 & \text { none } & 0\end{array}$




\section{Answers re-analysed}

Add various fraction-simplifying rules to $\mathcal{V}$, and

$$
\text { U2 } \sin a * \cos a=\frac{1}{2} \sin 2 * a \text {. }
$$

Table: Re-analysed answers: $\frac{\mathrm{d} \sin ^{2} 2 x}{\mathrm{~d} x}=2 \sin 4 x$

$\begin{array}{lclr}\text { No. } & \text { Student's answer } & \text { relation } & \text { Score } \\ \text { 1. } & 4 \sin 2 x \cos 2 x & \equiv \mathcal{U} & 1 \\ \text { 2. } & \frac{\mathrm{d} \sin ^{2} 2 x}{\mathrm{~d} x} & \equiv \mathcal{F} & 0 \\ \text { 3. } & 2 \sin 2 x \cos 2 x & \mathrm{~F} & \text { buggy } \\ \text { 4. } & 2 \times 2 \sin 2 x \cos 2 x & \equiv \mathcal{V} & 0.8 \\ \text { 5. } & 2 \sin 4 x & = & 1 \\ 6 . & 2 \sin 2 x \cos 2 x+2 \sin 2 x \cos 2 x & \equiv \mathcal{V} & 0.8 \\ \text { 7. } & x / 4-\sin (4 * x) / 8 & \text { none } & 0\end{array}$

Note that answer 5 is now precisely right. 


\section{Variations on a theme}

- We could have added rule $\mathrm{U} 2$ to the set $\mathcal{V}$, rather than to $\mathcal{U}$. This would then mean that $2 \sin 4 x$ was now right, but $4 \sin 2 x \cos 2 x$, although still mathematically correct, only scores 0.8 , since it is only equivalent to the right answer under venial rules. 


\section{Variations on a theme}

- We could have added rule $\mathrm{U} 2$ to the set $\mathcal{V}$, rather than to $\mathcal{U}$. This would then mean that $2 \sin 4 x$ was now right, but $4 \sin 2 x \cos 2 x$, although still mathematically correct, only scores 0.8 , since it is only equivalent to the right answer under venial rules.

- We could create a new class $\mathcal{V}^{\prime}$ of "weakly venial" rules between $\mathcal{U}$ and $\mathcal{V}$, with $\mathrm{U} 2$ in it, and say that $\equiv \mathcal{V}^{\prime}$ was worth, say, 0.9 rather than 0.8 . 


\section{Variations on a theme}

- We could have added rule $\mathrm{U} 2$ to the set $\mathcal{V}$, rather than to $\mathcal{U}$. This would then mean that $2 \sin 4 x$ was now right, but $4 \sin 2 x \cos 2 x$, although still mathematically correct, only scores 0.8 , since it is only equivalent to the right answer under venial rules.

- We could create a new class $\mathcal{V}^{\prime}$ of "weakly venial" rules between $\mathcal{U}$ and $\mathcal{V}$, with $\mathrm{U} 2$ in it, and say that $\equiv \overline{\mathcal{V}}^{\prime}$ was worth, say, 0.9 rather than 0.8 .

- The teacher could vary the approach over time, saying "from now on, I expect you to use trigonometric contraction where appropriate", and move $\mathrm{U} 2$ from $\mathcal{U}$ to $\mathcal{V}^{\prime}$, and maybe on to $\mathcal{V}$ after a couple of weeks. 


\section{Variations on a theme}

- We could have added rule $\mathrm{U} 2$ to the set $\mathcal{V}$, rather than to $\mathcal{U}$. This would then mean that $2 \sin 4 x$ was now right, but $4 \sin 2 x \cos 2 x$, although still mathematically correct, only scores 0.8 , since it is only equivalent to the right answer under venial rules.

- We could create a new class $\mathcal{V}^{\prime}$ of "weakly venial" rules between $\mathcal{U}$ and $\mathcal{V}$, with $\mathrm{U} 2$ in it, and say that $\equiv_{\mathcal{V}^{\prime}}$ ' was worth, say, 0.9 rather than 0.8 .

- The teacher could vary the approach over time, saying "from now on, I expect you to use trigonometric contraction where appropriate", and move $\mathrm{U} 2$ from $\mathcal{U}$ to $\mathcal{V}^{\prime}$, and maybe on to $\mathcal{V}$ after a couple of weeks.

- Indeed, one could imagine a stronger form of $\mathcal{V}$, which cost $50 \%$ of the marks. 
Conclusions 


\section{Conclusions}

- We can't let any algebra get at the student's input before we do! 


\section{Conclusions}

- We can't let any algebra get at the student's input before we do!

- This is going to be even more important as we develop tests like 'factor', 'express as partial fractions' etc. 


\section{Conclusions}

- We can't let any algebra get at the student's input before we do!

- This is going to be even more important as we develop tests like 'factor', 'express as partial fractions' etc.

- This formalism may actually help a teacher explain why, rather than just say "I expected you to". 


\section{Conclusions}

- We can't let any algebra get at the student's input before we do!

- This is going to be even more important as we develop tests like 'factor', 'express as partial fractions' etc.

- This formalism may actually help a teacher explain why, rather than just say "I expected you to".

- We do not preclude use of the full power of a computer algebra system - "the system thinks your answer is right, but you'd better get it marked manually". 
Future Work 
Future Work

- Implement it! (JHD has a preference for Axiom) 


\section{Future Work}

- Implement it! (JHD has a preference for Axiom)

- "However, if a specified simplification of an expression is desired, as is often the case in college algebra and precalculus courses, WeBWork cannot be used." [AMS] 


\section{Future Work}

- Implement it! (JHD has a preference for Axiom)

- "However, if a specified simplification of an expression is desired, as is often the case in college algebra and precalculus courses, WeBWork cannot be used." [AMS]

- This will require a blend of syntactic analysis and the techniques mentioned above. 


\section{References}

粗 J. Lewis and A. Tucker.

Report of the AMS First-Year Task Force.

Notices AMS, 56:754-760, 2009.

园 C. J. Sangwin.

STACK: making many fine judgements rapidly.

In CAME, 2007.

R.M. Young and T. O'Shea.

Errors in Children's Subtraction.

Cognitive Science, 5:153-177, 1981. 